Journal of Computer Science 3 (7): 523-527, 2007

ISSN 1549-3636

(C) 2007 Science Publications

\title{
Numerical Solutions of the Multispecies Predator-Prey Model by Variational Iteration Method
}

\author{
Khaled Batiha \\ Department of Computer Science, Irbid National University, Jordan
}

\begin{abstract}
The main objective of the current work was to solve the multispecies predator-prey model. The techniques used here were called the variational iteration method (VIM) and the Adomian decomposition method (ADM). The advantage of this work is twofold. Firstly, the VIM reduces the computational work. Secondly, in comparison with existing techniques, the VIM is an improvement with regard to its accuracy and rapid convergence. The VIM has the advantage of being more concise for analytical and numerical purposes. Comparisons with the exact solution and the fourth-order Runge-Kutta method (RK4) show that the VIM is a powerful method for the solution of nonlinear equations.
\end{abstract}

Key words: Variational iteration method (VIM), Adomian decomposition method (ADM), fourthorder Runge-Kutta method (RK4), multispecies predator-prey model

\section{INTRODUCTION}

The variational iteration method (VIM) was proposed by $\mathrm{He}^{[1]}$ initially with the aims to solve frontier physical problem. It has been applied to a wide class of deterministic and stochastic problems, linear and nonlinear, in physics, biology and chemical reactions etc. For nonlinear models, the method has shown reliable results in supplying analytical approximation that converges very rapidly ${ }^{[2-8]}$.

The Adomian decomposition method (ADM) was first envisioned by Adomian ${ }^{[9]}$ for solving a wide Range of problems whose mathematical models yield differential equation or system of differential equations $^{[10]}$.

The multispecies predator-prey equations (which it called also Lotka-Volterra equations) model the dynamic behavior of an arbitrary number of competitors $^{[11]}$. Though originally formulated to describe the time history of a biological system, this model find his application in a number of engineering fields such as simultaneous chemical and nonlinear control. In fact, the one predator one prey model is one of the most popular ones to demonstrate a simple nonlinear control system.

The purpose of this paper is to obtain approximate analytical solutions for multispecies predator-prey model and to make a numerical comparison between VIM, ADM and RK4 for solving the multispecies predator-prey model.
Variational iteration method: This method, which is a modified general Lagrange's multiplier method ${ }^{[12]}$, has been shown to solve effectively, easily and accurately a large class of nonlinear problems ${ }^{[4-8]}$. The main feature of the method is that the solution of a mathematical problem with linearization assumption is used as initial approximation or trial function, and then a more highly precise approximation at some special point can be obtained. This approximation converges rapidly to an accurate solution. To illustrate the basic concepts of the VIM, we consider the following nonlinear differential equation:

$$
L u+N u=g(x)
$$

where $L$ is a linear operator, $N$ is a nonlinear operator, and $g(x)$ is an inhomogeneous term. According to the VIM, we can construct a correction functional as follows:

$$
\begin{aligned}
& u_{n+1}(x)=u_{n}(x)+\int_{0}^{x} \lambda(s)\left[L u_{n}(s),\right. \\
& \left.+N \tilde{u}_{n}(s)-g(s)\right] d s,(n \geq 0)
\end{aligned}
$$

where $\lambda$ is a general Lagrangian multiplier ${ }^{[12]}$, which can be identified optimally via the variational theory, the subscript $n$ denotes the $n$ th-order approximation, $\tilde{u}_{n}$ is considered as a restricted Variation i.e. $\delta \tilde{u}_{n}=0$. The objective of this study is not a qualitative analysis of the rich dynamic behavior of the noted equations, but

Corresponding Author: Khaled Batiha, Department of Computer Science, Irbid National University, Jordan, P.O. Box: 2600 Irbid 21110 Jordan 
to present an accurate solution to the multispecies predator-prey model (Lotka-Volterra equations) for an arbitrary number of competitors, using decomposition method and variational iteration method.

\section{RESULTS AND DISCUSSION}

We now obtain numerical solutions of the multispecies predator-prey model. The VIM and ADM algorithms are coded in the computer algebra package Maple. The Maple environment variable Digits controlling the number of significant digits is set to 20 in all the calculations done in this study. Consider the general predator-prey model for an $m$ species system

$$
\frac{d R_{i}}{d t}=R_{i}\left[b_{i}+\sum_{j=1}^{m} a_{i j} R_{j}\right]
$$

, $i=1,2, \ldots, m$.

These equations represent multispecies predator-prey cases, cf. $^{[13,14]}$

One species:

In this case Eq. (3) reduced to one species competing for a given finite source of food:

$\frac{d R}{d t}=R(b+a R)$,

where $a<0, b>0$ are constants and $R(0)>0$. This equation has an exact solution

$$
\begin{aligned}
& R(t)=\frac{b e^{b t}}{\frac{b+a R(0)}{R(0)}-a e^{b t}} \text { for } b \neq 0, \\
& R(t)=\frac{R(0)}{1-a R(0) t} \text { for } b=0
\end{aligned}
$$

To solve equation (4) by means of He's variational iteration method, we construct a correction functional,

$$
\begin{aligned}
& R_{n+1}(t)=R_{n}(t)+\int_{0}^{t} \lambda(s)\left[\frac{d R_{n}(s)}{d s}\right. \\
& \left.--b R_{n}(s)-a \tilde{R}_{n}^{2}(s)\right] d s
\end{aligned}
$$

where $\tilde{R}_{n}$ is considered as restricted variations, which mean $\delta \tilde{R}_{n}=0$. Its stationary conditions can be obtained as follows

$$
1+\lambda(t)=0, \lambda^{\prime}(s)+\left.b \lambda(s)\right|_{s=t}=0,
$$

The Lagrange multipliers, therefore, can be identified as $\lambda(s)=-e^{-b(s-t)}$ and the following variational iteration formula can be obtained by

$$
\begin{aligned}
& R_{n+1}(t)=R_{n}(t)-\int_{0}^{t} \frac{e^{-b s}}{e^{-b t}}\left[\frac{d R_{n}(s)}{d s}\right. \\
& \left.-b R_{n}(s)-a R_{n}^{2}(s)\right] d s,
\end{aligned}
$$

which results,

$$
\begin{aligned}
& R_{1}(t)=R_{0}(t)-\int_{0}^{t} \frac{e^{-b s}}{e^{-b t}}\left[\frac{d R_{0}(s)}{d s}-b R_{0}(s)-a R_{0}^{2}(s)\right] d s, \\
& R_{2}(t)=R_{1}(t)-\int_{0}^{t} \frac{e^{-b s}}{e^{-b t}}\left[\frac{d R_{1}(s)}{d s}-b R_{1}(s)-a R_{1}^{2}(s)\right] d s, \\
& \vdots
\end{aligned}
$$

We can take the linearized solution $R(t)=c e^{b t}$ as the initial approximation $R(0)$, then we get

$R_{1}(t)=c e^{b t}+\frac{a c^{2} e^{b t}\left(e^{b t}-1\right)}{b}$

the condition $R(0)=0.1$ gives us $c=0.1$. Thus

$$
R_{1}(t)=0.1 e^{b t}+\frac{0.001 a e^{b t}\left(e^{b t}-1\right)}{b}
$$

In the same manner, the rest of the components of the iteration formulae (9) can be obtained using the Maple Package.

To solve equation (4) by the ADM with initial condition $R(0)=0.1$, using the inverse operator $L^{-1}(\cdot)=\int_{0}^{t}(\cdot) d t$ we get:

$R_{0}=R(0), R_{n+1}(t)=\int_{0}^{t}\left(b R_{n}+a A_{1, n}\right) d t$

$n \geq 0$,

where the Adomian polynomial

$$
A_{1, n}=\sum_{k=0}^{n} R_{k} R_{n-k}
$$

which results

$$
\begin{aligned}
& R_{1}(t)=\int_{0}^{t}\left(b R_{0}+a\left(R_{0}^{2}\right)\right) d t \\
& R_{1}(t)=\int_{0}^{t}\left(b R_{0}+a\left(R_{0} R_{1}+R_{1} R_{0}\right)\right) d t \\
& \vdots \\
& \text { Table 1: } \quad \text { Numerical comparison when } \mathrm{b}=1, a=-3, R(0)=0.1
\end{aligned}
$$


J. Computer Sci., 3 (7): 523-527, 2007

\begin{tabular}{llll}
\hline $\mathrm{t}$ & Exact solution & $\mathrm{ADM}, \phi_{3}$ & 2-iterate VIM \\
\hline 0.0 & 0.1000000000 & 0.1000000000 & 0.1000000000 \\
0.2 & 0.1145329055 & 0.1145600000 & 0.1145545368 \\
0.4 & 0.1300011386 & 0.1302400000 & 0.1302589889 \\
0.6 & 0.1461628997 & 0.1470400000 & 0.1474445088 \\
0.8 & 0.1627259130 & 0.1649600000 & 0.1671263185 \\
1.0 & 0.1793671754 & 0.1840000000 & 0.1915249307 \\
\hline
\end{tabular}

Two species:

The predator-prey model for this case takes the following form

$$
\begin{aligned}
& \frac{d R_{1}}{d t}=R_{1}\left(b_{1}+a_{11} R_{1}+a_{12} R_{2}\right) \\
& \frac{d R_{2}}{d t}=R_{2}\left(b_{2}+a_{21} R_{1}+a_{22} R_{2}\right)
\end{aligned}
$$

Equations (14) and (15) model two species competing for a common ecological niche.

To solve equation (14) and (15) by means of He's variational iteration method, we construct a correction function,

$$
\begin{aligned}
& R_{1, n+1}(t)=R_{1, n}(t)+\int_{0}^{t} \lambda_{1}(s)\left[\frac{d R_{1, n}(s)}{d s}\right. \\
& \left.-b_{1} R_{1, n}(s)-a_{11} \tilde{R}_{1, n}^{2}(s)-a_{12} \tilde{R}_{1, n}(s) \tilde{R}_{2, n}(s)\right] d s \\
& R_{2, n+1}(t)=R_{2, n}(t)+\int_{0}^{t} \lambda_{2}(s)\left[\frac{d R_{2, n}(s)}{d s}\right. \\
& \left.-b_{2} R_{2, n}(s)-a_{22} \tilde{R}_{2, n}^{2}(s)-a_{21} \tilde{R}_{2, n}(s) \tilde{R}_{1, n}(s)\right] d s
\end{aligned}
$$

where $\tilde{R}_{i, n}$ is considered as restricted variations, which mean $\delta \tilde{R}_{i, n}=0$. Its stationary conditions can be obtained as follows

$$
\begin{aligned}
& 1+\lambda_{1}(t)=0, \lambda_{1}{ }^{\prime}(s)+\left.b_{1} \lambda_{1}(s)\right|_{s=t}=0, \\
& 1+\lambda_{2}(t)=0, \lambda_{2}{ }^{\prime}(s)+\left.b_{2} \lambda_{2}(s)\right|_{s=t}=0
\end{aligned}
$$

The Lagrange multipliers, therefore, can be identified as $\lambda_{1}(s)=-e^{-b_{1}(s-t)}$ and $\lambda_{2}(s)=-e^{-b_{2}(s-t)}$, and the following variational iteration formula can be obtained by

$$
\begin{aligned}
& R_{1, n+1}(t)=R_{1, n}(t)+\int_{0}^{t} \frac{e^{-b_{1} s}}{e^{-b_{1} t}}\left[\frac{d R_{1, n}(s)}{d s}\right. \\
& \left.-b_{1} R_{1, n}(s)-a_{11} R_{1, n}^{2}(s)-a_{12} R_{1, n}(s) R_{2, n}(s)\right] d s
\end{aligned}
$$

$$
\begin{aligned}
& R_{2, n+1}(t)=R_{2, n}(t)+\int_{0}^{t} \frac{e^{-b_{2} s}}{e^{-b_{2} t}}\left[\frac{d R_{2, n}(s)}{d s}-b_{2} R_{2, n}\right. \\
& \left.(s)-a_{22} R_{2, n}^{2}(s)-a_{21} R_{2, n}(s) R_{1, n}(s)\right] d s,
\end{aligned}
$$

which results

$$
\begin{aligned}
& R_{1,1}(t)=R_{1,0}(t)+\int_{0}^{t} \frac{e^{-b_{1} s}}{e^{-b_{1} t}}\left[\frac{d R_{1,0}(s)}{d s}-b_{1} R_{1,0}(s)\right. \\
& \left.-a_{11} R_{1,0}^{2}(s)-a_{12} R_{1,0}(s) R_{2,0}(s)\right] d s \\
& R_{2,2}(t)=R_{2,1}(t)+\int_{0}^{t} \frac{e^{-b_{2} s}}{e^{-b_{2} t}}\left[\frac{d R_{2,1}(s)}{d s}-b_{2} R_{2,1}(s)\right. \\
& \left.-a_{22} R_{2,1}^{2}(s)-a_{21} R_{2,1}(s) R_{1,1}(s)\right] d s
\end{aligned}
$$

$\vdots$

We can take the linearized solution $R_{1}(t)=c_{1} e^{b_{1} t}$ and $R_{2}(t)=c_{2} e^{b_{2} t} \quad$ as the initial approximation. The conditions $R_{1}(0)=4$ and $R_{2}(0)=10$ gives $c_{1}=4$ and $_{c_{2}}=10$. Thus

$$
\begin{aligned}
& R_{1,1}(t)=c_{1} e^{b_{1} t}+\frac{c_{1} e^{b_{1} t}\left(-a_{11} c_{1} b_{2}-a_{12} c_{2} b_{1}+a_{11} c_{1} e^{b_{1} t} b_{2}+a_{12} c_{2} e^{b_{1} t} b_{1}\right)}{b_{1} b_{2}} \\
& R_{2,1}(t)=c_{2} e^{b_{2} t}+\frac{c_{2} e^{b_{2} t}\left(-a_{21} c_{1} b_{2}-a_{22} c_{2} b_{1}+a_{21} c_{1} e^{b_{1} t} b_{2}+a_{22} c_{2} e^{b_{2 t} t} b_{1}\right)}{b_{1} b_{2}}
\end{aligned}
$$

Again, the rest of the components of the iteration formulae (20) and (21) can be obtained using the Maple Package.

To solve equation (14) and (15) by the ADM with initial condition $R_{1}(0)=4$ and $R_{2}(0)=10$, applying the inverse operator $L^{-1}(\cdot)=\int_{0}^{t}(\cdot) d t$ we get:

$R_{1,0}=R_{1}(0), R_{2,0}=R_{2}(0)$

$R_{1, n+1}(t)=\int_{0}^{t}\left(b_{1} R_{1, n}+a_{11} A_{1, n}+a_{12} A_{1,2, n}\right) d t, n \geq 0$,

$R_{2, n+1}(t)=\int_{0}^{t}\left(b_{2} R_{2, n}+a_{21} A_{2,1, n}+a_{22} A_{2, n}\right) d t, n \geq 0$,

where the Adomian polynomials are

$A_{1, n}=\sum_{k=0}^{n} R_{1, k} R_{1, n-k}, A_{1,2, n}=\sum_{k=0}^{n} R_{1, k} R_{2, n-k}$,

$A_{2, n}=\sum_{k=0}^{n} R_{2, k} R_{2, n-k}, A_{2,1, n}=\sum_{k=0}^{n} R_{2, k} R_{1, n-k}$,

which results

$R_{1,1}(t)=\int_{0}^{t}\left(b_{1} R_{1,0}+a_{11}\left(R_{1,0} R_{1,0}\right)+a_{12}\left(R_{1,0} R_{2,0}\right)\right) d t$ 
J. Computer Sci., 3 (7): 523-527, 2007

\begin{tabular}{|c|c|c|c|c|c|c|}
\hline \multirow[t]{2}{*}{$\mathrm{t}$} & \multicolumn{2}{|l|}{$\mathrm{ADM}, \phi_{3}$} & \multicolumn{2}{|l|}{ 2-iterat VIM } & \multicolumn{2}{|l|}{ RK4 } \\
\hline & $\mathrm{R}_{1}$ & $\mathrm{R}_{2}$ & $\mathrm{R}_{1}$ & $\mathrm{R}_{2}$ & $\mathrm{R}_{1}$ & $\mathrm{R}_{2}$ \\
\hline 0.0 & 4.000000000 & 10.00000000 & 4.000000000 & 10.00000000 & 4.000000000 & 10.00000000 \\
\hline 0.2 & 4.066362522 & 10.13348966 & 4.066363553 & 10.13349043 & 4.066363454 & 10.13349028 \\
\hline 0.4 & 4.133610086 & 10.26835866 & 4.133618314 & 10.26836476 & 4.133617490 & 10.26836347 \\
\hline 0.6 & 4.201742694 & 10.40460698 & 4.201770394 & 10.40462736 & 4.201767478 & 10.40462287 \\
\hline 0.8 & 4.270760346 & 10.54223462 & 4.270825823 & 10.54228247 & 4.270818626 & 10.54227146 \\
\hline 1.0 & 4.340663040 & 10.68124160 & 4.340790588 & 10.68133427 & 4.340775941 & 10.68131190 \\
\hline
\end{tabular}

$$
\begin{aligned}
& R_{1,2}(t)=\int_{0}^{t}\left(b_{1} R_{1,1}+a_{11}\left(R_{1,0} R_{1,1}+R_{1,1} R_{1,0}\right)+a_{12}\left(R_{1,0} R_{2,1}+R_{1,1} R_{2,0}\right)\right) d t \\
& \vdots \\
& R_{2,1}(t)=\int_{0}^{t}\left(b_{2} R_{2,0}+a_{21}\left(R_{2,0} R_{1,0}\right)+a_{22}\left(R_{2,0} R_{2,0}\right)\right) d t \\
& R_{2,2}(t)=\int_{0}^{t}\left(b_{1} R_{2,1}+a_{21}\left(R_{2,0} R_{1,1}+R_{2,1} R_{1,1}\right)+a_{12}\left(R_{2,0} R_{2,1}+R_{2,1} R_{2,0}\right)\right) d t \\
& \vdots
\end{aligned}
$$

We compare with the exact solution (only for one species), ADM, VIM and RK4. Table 1 shows the case of one species comparison between the 2-iterate of VIM, 3 -term in the series of ADM and exact solution for $b=$ $1, a=-3$ and $R(0)=0.1$. Table 2 shows two species with comparison between the 2-iterate of VIM, 3-term in the series of ADM and RK4 with $h=0.001, b_{1}=0.1$, $a_{11}=-0.0014, a_{12}=-0.0012, b_{2}=0.08, a_{21}=-0.0009$, $a_{22}=-0.001, R_{1}(0)=4$ and $R_{2}(0)=10$. There are some important points to make here. First, the VIM provides the solutions in terms of convergent series with easily computable components. Second, it is clear and remarkable that approximate solutions using VIM are in good agreement. Third, the VIM technique requires less computational work than ADM and other existing approaches while supplying quantitatively reliable results. The results presented in Table 1 and Table 2 clearly shows that good accuracy of VIM.

\section{CONCLUSION}

In this paper, variation iteration method (VIM) has been successfully employed to obtain the approximate solution of the multispecies predator-prey model. We compare between the exact solution (only in one species), VIM, ADM and RK4 applied to multispecies predator-prey model. Comparisons with the ADM and the RK4 show that the VIM is a powerful method for the solution of nonlinear equations. The advantage of the VIM over the ADM is that there is no need for the evaluation of the Adomian polynomials and the advantage over the RK4 method is that the VIM gives continuous solutions. The VIM was used in a direct way without using linearization, perturbation or restrictive assumptions. The VIM provides more realistic series solutions that converge very rapidly in real physical problems.

\section{REFERENCES}

1. He, J.H., 1997. A new approach to nonlinear partial differential equations, Nonlinear Sci. Numer. Simu., 2: 230-235.

2. He, J.H., 1999. Variational iteration method--a kind of non-linear analytical technique: some examples, Intern. J. Non-Linear Mech. 34: 699708.

3. He, J.H., 2003. A simple perturbation approach to Blasius equation, Appl. Math. Comput., 140: 217222.

4. He, J.H., 2006. Variational iteration method- some recent results and new interpretations, J. Comput. Appl. Math., in press.

5. Soliman, A.A., 2006. A numerical simulation and explicit solutions of KdV-Burger's and Lax's seventh-order KdV equations, Chaos, Solitons and Fractals, in press.

6. Abulwafa, E.M., Abdou, M.A. and Mahmoud, A.A., 2006. The solution of nonlinear coagulation problem with mass loss, Chaos, Solitons and Fractals, in press.

7. Abbasbandy, S., 2006. A new application of He's variational iteration method for quadratic Riccati differential equation by using adomian's polynomials, J. Comput. Appl. Math., in press.

8. Wazwaz, A.M., 2006. The variational iteration method for rational solutions for $\mathrm{KdV}, \quad K(2,2)$, Burgers, and cubic Boussinesq equations, J. Comput. Appl. Math., in press.

9. Adomian. G, (1994). Solving frontier problems of physics: the decomposition method. Boston: Kluwer Academic. 
10. Chen W and Z. Lu, 2004. An algorithm for Adomian decomposition method. Appl. Math. Comput., 159: 221-235.

11. Hofbauer, J and K. Sigmund, (1988) The theory of evolution and dynamical systems, Cambridge University press, London.

12. Inokuti, M., H. Sekine and T. Mura, 1978. General use of the Lagrange multiplier in nonlinear mathematical physics. In: Nemat-Nassed S, editor. Variational method in the mechanics of solids. Pergamon press, 156-162.
13. Olek S., 1994. An accurate solution to the multispecies Lotka-Volterra equations. SIAM Review, 36: 480-488.

14. May, R.M. and Leonard W.J., 1975. Nonlinear aspects of competition between three species. SIAM J. Appl. Math., 29: 243-253. 